\title{
An effective thermal-parametrization theory for the slow-light dynamics in a Doppler-broadened electomagnetically induced transparency medium
}

\author{
Shih-Wei Su, ${ }^{1}$ Yi-Hsin Chen, ${ }^{1}$ Shih-Chuan Gou, ${ }^{2,}{ }^{*}$ and Ite A. Yu ${ }^{1, \dagger}$ \\ ${ }^{1}$ Department of Physics, National Tsing Hua University, Hsinchu 30013, Taiwan \\ ${ }^{2}$ Department of Physics, National Changhua University of Education, Changhua 50058, Taiwan
}

(Dated: Feb 25, 2011)

\begin{abstract}
We model the effects of atomic thermal motion on the propagation of a light pulse in an electromagnetically induced transparency medium by introducing a set of effectively temperature-dependent parameters, including the Rabi frequency of the coupling field, optical density and relaxation rate of the ground state coherence, into the governing equations. The validity of this effective theory is verified by the close agreement between the theoretical results and the experimental data.
\end{abstract}

PACS numbers: 42.50.Gy, 32.80.Qk, 02.60.Jh

\section{INTRODUCTION}

The ability of controlling light is crucial to the fulfillment of quantum communications and all their practical applications. It has been demonstrated recently that a light pulse can be slowed down [1, 2] and even stored in media [3, 4, by using the effect of electromagnetically induced transparency (EIT), a nonlinear optical phenomenon that renders an opaque medium transparent by irradiating it with an electromagnetic field [5] . Subsequent studies indicate that the slow light (SL) effect can greatly enhance optical nonlinearity at the low light level [6] , and has potential applications in the development of low-light-level and single-photon devices, such as the all-optical switches [7] and quantum phase gates based on the cross-phase modulation scheme [8, 9] . The novelty of EIT thus provides a new fashion to manipulate the behavior of light, and has attracted a great deal of research interest.

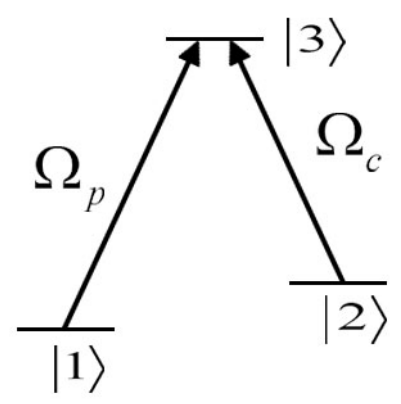

FIG. 1: (Color online) The energy levels of a 3-level atom of $\Lambda$-type. The transitions $|1\rangle \leftrightarrow|3\rangle$ and $|2\rangle \leftrightarrow|3\rangle$ are driven by laser fields with Rabi frequencies $\Omega_{p}$ and $\Omega_{c}$ correspondingly.

The prototype of EIT systems consists of a weak probe

*Electronic address: scgou@cc.ncue.edu.tw

${ }^{\dagger}$ Electronic address: yu@phys.nthu.edu.tw beam of central frequency $\omega_{p}$ and a strong coupling beam of frequency $\omega_{c}$ interacting with a three-level atom, as shown in Fig.1. The dynamics of the slowly varying density matrix elements in the weak probe limit are described by the optical Bloch equation [10]

$$
\begin{gathered}
\frac{\partial \rho_{12}}{\partial t}=-\left[i\left(\Delta_{p}-\Delta_{c}\right)+\gamma\right] \rho_{12}-\frac{i \Omega_{c}}{2} \rho_{13} \\
\frac{\partial \rho_{13}}{\partial t}=-\left(i \Delta_{p}+\frac{\Gamma}{2}\right) \rho_{13}-\frac{i \Omega_{c}^{*}}{2} \rho_{12}-\frac{i \Omega_{p}^{*}}{2}
\end{gathered}
$$

where $\Omega_{p}$ and $\Omega_{c}$ denote the Rabi frequencies driving the transitions $|1\rangle \leftrightarrow|3\rangle$ and $|2\rangle \leftrightarrow|3\rangle$ respectively; $\gamma$ is the relaxation rate of the ground-state coherence, and $\Gamma$ is the spontaneous decay rate of the excited state $|3\rangle$. The detunings $\Delta_{p}$ and $\Delta_{c}$ are defined by $\Delta_{p}=\omega_{31}-\omega_{p}$ and $\Delta_{c}=\omega_{32}-\omega_{c}$, where $\omega_{i j}$ denotes the transition frequency between the energy levels $|i\rangle$ and $|j\rangle$. The propagation of the probe pulse is described by the Maxwell-Schrödinger equation

$$
\frac{1}{c} \frac{\partial \Omega_{p}(z, t)}{\partial t}+\frac{\partial \Omega_{p}(z, t)}{\partial z}=i \eta \rho_{31}
$$

where $\eta$ measures the opacity per unit length of the medium and is related to the optical density of the medium by $d^{o p t}=2 L \eta / \Gamma$ ( $L$ is the medium length). Here $\rho_{31}$ corresponds to the slowly varying amplitude of the optical coherence of the probe transition.

We note that a decay constant $\gamma$ is introduced in eq. (1) to account for the relaxation of $\rho_{21}$. Phenomenologically, we can write $\gamma=\gamma_{T}+\gamma_{0}$, where $\gamma_{T}$ represents the effects originating from the thermal random motion of atoms and $\gamma_{0}$ represents other intrinsic effects, such as the linewidth and frequency fluctuations of the laser fields, the stray magnetic field, and the collisions between atoms, etc. Except the demonstration of SL in BoseEinstein condensate [11, 12], most EIT experiments have so far been carried out using either laser-cooled atoms or hot atoms, with temperatures widely ranging from milliKelvins to room temperature [13, 14]. It is recognized 
that the randomized Doppler shifts stemming from the thermal motion of atoms is the predominating mechanism of decoherence at finite temperatures. Since the temperature as an independent variable does not enter eqs.(1) - (3), $\gamma_{T}$ is bound to depend implicitly on the temperature and can only be determined by numerical fitting with the experimental data.

In order to gain more insight into the dynamical properties of an EIT medium at finite temperatures, it is desirable to take the atom's motion into account by statistical approach. Javan et. al. suggested that the macroscopic polarization, which serves as a response to the randomized frequency shift, should be obtained by taking average over all possible velocities characterized by the Maxwell-Boltzmann distribution [15, 16] . It is expected that such an averaging process smears the atomic coherence and thus contribute largely to the relaxation of $\rho_{12}$. More recently, the dynamics of SL, light storage and stationary light pulse in finite-temperature EIT media are studied in [17, 18, Wu et. al. 17, have developed another method to solve the dynamics of stationary light pulses in a cold atomic cloud by solving the Maxwell-Liouville equations which contain the higher order spin and optical coherence and the Doppler effect arising from the atomic thermal motion. By using the gauge-invariance of Schrödinger equations under the Galilean transformation, $\mathrm{Su}$ et. al. [18] have developed a numerical scheme to study the dynamics of SL and light storage in a Doppler-broadened EIT medium, in which the effects of the atom's external degrees of freedom are fully considered.

In this paper, we investigate the dynamics of SL in a Doppler-broadened EIT based on the formalism of optical Bloch equations. The central idea of this work is to treat the atom as a stationary object but otherwise introduce some temperature-dependent parameters which are responsible for the manifestation of thermal effects. Specifically, we analyze the output probe pulse under the influence of the atomic thermal motion by endowing the coupling field, the optical density and the phenomenological decay constant with temperature dependence, namely, we let $\Omega_{c} \rightarrow \bar{\Omega}_{c}(T), \eta \rightarrow \bar{\eta}(T), \gamma \rightarrow \bar{\gamma}(T)$ for a stationary EIT medium. Derivation of these effective parameters will be described in the following.

\section{FORMALISM}

To begin with, we assume that the shape distortion of the output probe pulse can be treated as a superposition of plane waves of different frequencies propagating through the highly dispersive and slightly absorptive EIT medium with length $L$. Each output plane wave attains a factor of $e^{i 2 \pi L\left(n_{R}+i n_{I}\right) / \lambda}$ while propagating inside the medium, where $n_{I}$ and $n_{R}$ are the real and imaginary parts of the refractive index of the medium, which lead to the attenuation and phase shift of the output plane waves, respectively. Note that the refractive index is re- lated to $\rho_{31}$ by $2 \pi\left(n_{R}+i n_{I}\right) / \eta \lambda=\rho_{31} / \Omega_{p}[19]$.

In the weak probe limit, the steady state solution of $\rho_{31}$ of the $\Lambda$-type EIT system is given by [19]

$$
\frac{\rho_{31}}{\Omega_{p}}=\frac{\Delta_{p}-\Delta_{c}+i \gamma}{\Omega_{c}^{2} / 2-2\left(\Delta_{p}+i \Gamma / 2\right)\left(\Delta_{p}-\Delta_{c}+i \gamma\right)},
$$

which can be expanded as a power series of $\omega$ :

$$
\frac{\rho_{31}}{\Omega_{p}}=\sum b^{(i)}\left(\omega-\omega_{p}\right)^{i},
$$

where $\omega_{p}$ is the center frequency of the probe pulse. It has been shown that for an input Gaussian probe pulse with a width $\tau_{0}$,

$$
\Omega_{p, i n}(t)=\Omega_{p 0} e^{-t^{2} / \tau_{0}^{2}},
$$

the output probe pulse will take the form [19.

$$
\Omega_{p, \text { out }}(t)=\Omega_{p 0}\left(\frac{\tau_{0}}{\tau_{w}}\right) e^{-\beta-\left(t-\tau_{d}\right)^{2} / \tau_{w}^{2}}
$$

where $\beta=\operatorname{L\eta Im}\left[b^{(0)}\right], \tau_{d}=\operatorname{L\eta Re}\left[b^{(1)}\right]$ and $\tau_{w}=$ $\sqrt{\tau_{0}^{2}+4 \operatorname{Lim}\left[b^{(2)}\right]}$, corresponding to the attenuation constant, delay time and the broadened width of the output probe pulse, respectively. In deriving eq.(7), we retain the power series in eq.(5) merely up to the quadratic terms and set $\Delta_{p}=\Delta_{c}$. Under the approximations of strong coupling field, $\Omega_{c}^{2} \gg \gamma \Delta_{p}$ and $\Omega_{c}^{2} \gg \gamma \Gamma, b^{(0)}$ and $b^{(2)}$ are purely imaginary numbers and $b^{(1)}$ is a real number. The above conditions for achieving eq.(7) are well justified in the EIT related experiments since the EIT bandwidth is proportional to $\Omega_{c}^{2} / \Gamma$ and the frequency bandwidth of the probe pulse limits the maximum value of $\left(\omega-\omega_{p}\right)$. Note that eq. (7) is obtained based on the assumption that all atoms are kept fixed. Realistically, the atoms move freely with a velocity distribution obeying the Maxwell-Boltzmann statistics at finite temperatures. To reconcile these two contradictory situations, eqs.(1)(2) should be understood as the motion equations seen by any observer fixed on the moving atoms. To incorporate the Doppler shift into eq.(4) in an uncomplicated way, we shall assume that the probe pulse propagates along the major-axis of the atomic cloud and the coupling field is applied with an angle $\theta$ with respect to the major-axis. We also assume that the center frequencies of coupling and probe fields are on two-photon resonance in the laboratory frame, namely, $\Delta_{p}=\Delta_{c}$. Hence, for an atom moving with a velocity $\mathbf{v}=\mathbf{v}_{\perp}+\mathbf{v}_{z}$ in the non-relativistic limit, where $\mathbf{v}_{z}$ is the velocity along the major-axis and $\mathbf{v}_{\perp}$ is the velocity in the transverse direction, it experiences the Doppler shifts so that $\Delta_{p} \rightarrow \Delta_{p}-k_{p} v_{z}$ and $\Delta_{c} \rightarrow \Delta_{c}-\left(k_{c} v_{z} \cos \theta+k_{c} v_{\perp} \sin \theta\right)$ [20]. As a result, the condition of two-photon resonance does not hold generally, and the two-photon detuning, $\delta=\Delta_{p}-\Delta_{c}$, is maximized when $\theta=\pi$. 
In the presence of Doppler shifts, all $b^{(i)}$ in eq. (5) now turns out to be velocity-dependent, i.e., $b^{(i)} \rightarrow$ $\tilde{b}^{(i)}\left(v_{z}, v_{\perp}\right)$. Statistically, $b^{(i)}$ can be replaced by an effective coefficient obtained by taking the ensemble average of $\tilde{b}^{(i)}\left(v_{z}, v_{\perp}\right)$ over the Maxwell-Boltzmann velocity distribution at a given temperature $T$,

$$
\tilde{b}^{(i)}(\mathbf{v}) \rightarrow \bar{b}^{(i)}(T)=\frac{1}{\pi v_{s}^{2}} \int d v_{z} d v_{\perp} \tilde{b}^{(i)}(\mathbf{v}) e^{-\mathbf{v}^{2} / v_{s}^{2}},
$$

where $v_{s}=\sqrt{2 k_{B} T / m}$ is the one-dimensional root-meansquare velocity. It should be noted that the ensembleaveraging process in eq. (8) is liable to the relaxation of $\rho_{21}$ owing to the atomic thermal motion. To be consistent, in each $\tilde{b}^{(i)}(v)$, we let $\gamma_{T}=0$ and keep $\gamma_{0}$ in $\gamma$ while evaluating the coefficients $\bar{b}^{(i)}(T)$. Our effective theory is formulated based on the above ensemble-averaging process, in which the analytical forms of $\bar{\Omega}_{c}(T), \bar{\eta}(T)$ and $\bar{\gamma}(T)$ are derived. The basic idea relies on the presumption that, either the stationary medium characterized by $\bar{\Omega}_{c}(T), \bar{\eta}(T)$ and $\bar{\gamma}(T)$ or the Doppler-broadened medium characterized by the temperature-independent $\Omega_{c}, \eta$ and $\gamma_{0}$, should lead to the same attenuation constant, delay time and the broadened width of the output probe pulse, namely,

$$
\begin{gathered}
\frac{\beta}{L}=\left.\eta \bar{b}^{(0)}(T)\right|_{\gamma_{0}, \Omega_{c}}=\left.\bar{\eta} b^{(0)}\right|_{\bar{\gamma}, \bar{\Omega}_{c}}, \\
\frac{\tau_{d}(T)}{L}=\left.\eta \bar{b}^{(1)}(T)\right|_{\gamma_{0}, \Omega_{c}}=\left.\bar{\eta} b^{(1)}\right|_{\bar{\gamma}, \bar{\Omega}_{c}},
\end{gathered}
$$

and

$$
\frac{\tau_{w}^{2}(T)-\tau_{0}^{2}}{4 L}=\left.\eta \bar{b}^{(2)}(T)\right|_{\gamma_{0}, \Omega_{c}}=\left.\bar{\eta} b^{(2)}\right|_{\bar{\gamma}, \bar{\Omega}_{c}} .
$$

We evaluate eqs.(9) - (11) by making use of eqs.(4) and (8), together with the assumption $\Delta_{p}=\Delta_{c}=0$ for a stationary medium, and this yields

$$
\begin{aligned}
& \int d^{2} v \frac{\left[h(\mathbf{v}, \theta)+i \gamma_{0}\right] e^{-v^{2} / v_{s}^{2}}}{\Omega_{c}^{2} / 2-2\left[h(\mathbf{v}, \theta)+i \gamma_{0}\right] G\left(v_{z}\right)} \\
= & \frac{\bar{\eta} \pi v_{s}^{2}}{\eta} \frac{\bar{\gamma}}{\bar{\Omega}_{c}^{2} / 2+\Gamma \bar{\gamma}}, \\
& \int d^{2} v \frac{\Omega_{c}^{2} / 2+\left[h(\mathbf{v}, \theta)+i \gamma_{0}\right]^{2} e^{-v^{2} / v_{s}^{2}}}{\left[\Omega_{c}^{2} / 2-2\left[h(\mathbf{v}, \theta)+i \gamma_{0}\right] G\left(v_{z}\right)\right]^{2}} \\
= & \frac{\bar{\eta} \pi v_{s}^{2}}{\eta} \frac{\bar{\Omega}_{c}^{2} / 2-2 \bar{\gamma}^{2}}{\left(\bar{\Omega}_{c}^{2} / 2+\Gamma \bar{\gamma}\right)^{2}},
\end{aligned}
$$

and

$$
\begin{aligned}
& \int d^{2} v \frac{4 h^{3}(\mathbf{v}, \theta)+\Omega_{c}^{2}\left[2 h(\mathbf{v}, \theta)+G\left(v_{z}\right)\right]+g(\mathbf{v}, \theta)}{\left[\Omega_{c}^{2} / 2-2\left[h(\mathbf{v}, \theta)+i \gamma_{0}\right] G\left(v_{z}\right)\right]^{3}} e^{-v^{2} / v} \\
= & \frac{\bar{\eta} \pi v_{s}^{2}}{\eta} \frac{\Gamma \bar{\Omega}_{c}^{2} / 2-4 \bar{\gamma}^{3}+2 \bar{\gamma} \bar{\Omega}_{c}^{2}}{\left(\bar{\Omega}_{c}^{2} / 2+\Gamma \bar{\gamma}\right)^{3}}
\end{aligned}
$$

where $h(\mathbf{v}, \theta)=k v_{\perp} \sin \theta-k v_{z}(1-\cos \theta), G\left(v_{z}\right)=$ $-k v_{z}+i \Gamma / 2$ and $g(\mathbf{v}, \theta)=12 \gamma_{0} h(\mathbf{v}, \theta)\left[i h(\mathbf{v}, \theta)-\gamma_{0}\right]+$ $2 i \gamma_{0} \Omega_{c}^{2}$. To further simplify the last three equations, we assume $|\sin \theta| \ll 1$ together with the assumptions $\Omega_{c}^{2} \gg \gamma_{0} \Gamma, \bar{\Omega}_{c}^{2} \gg \bar{\gamma} \Gamma, \Omega_{c}^{2} \gg \gamma_{0} \Delta_{p}, \Gamma \gg \bar{\gamma}$ and $k_{p} \approx k_{c}=k$ which are the typical conditions for the EIT experiments. The condition $|\sin \theta| \ll 1$ is generally true, since so far all EIT experiments have used either the nearly co-propagating $(\theta \approx 0)$ or counter-propagating $(\theta \approx \pi)$ geometry. In our experiments, the central wavelength of the laser beam is $780 \mathrm{~nm}, \gamma_{0} \simeq 5 \times 10^{-4} \Gamma$, $\bar{\gamma} \simeq 2 \times 10^{-3} \Gamma, \Delta_{p}=0$, and $0.6 \Gamma \leq \Omega_{c} \leq 0.9 \Gamma$, where the spontaneous decay rate of the excited state is $\Gamma=2 \pi \times 5.9 \mathrm{MHz}$. Obviously, the forementioned assumptions are valid and thus the effective parameters can now be solved as

$$
\begin{aligned}
& \bar{\Omega}_{c}(T) \\
= & \Omega_{c}\left\{1+4\left[2 f(\theta)-\frac{4+5 f(\theta)}{\Gamma^{2}} \Omega_{c}^{2}\right] x^{2}+\cdots\right\}, \\
& \bar{\eta}(T) \\
= & \eta\left\{1+8\left[f(\theta)-\frac{2+4 f(\theta)}{\Gamma^{2}} \Omega_{c}^{2}\right] x^{2}+\cdots\right\},
\end{aligned}
$$

and

$$
\bar{\gamma}(T)=\gamma_{0}+4 f(\theta) \frac{\Omega_{c}^{2}}{\Gamma} x^{2}+\cdots,
$$

where $f(\theta)=1-\cos \theta$ and $x=\sin (\theta / 2) k v_{s} \Gamma / \sqrt{2} \Omega_{c}^{2}$, which is the ratio of the effective Doppler width to that of the EIT window. In eq. 117), the leading term of $\bar{\gamma}(T)$ is the ground-state relaxation rate originating from the intrinsic effects and the second term is resulted from the Doppler shifts. When the probe and coupling fields are nearly co-propagating, i.e. $\theta \simeq 0$, the Doppler effect is considerably suppressed, and this is why the EIT experiments at room temperatures are always studied in the co-propagating geometry 13 . On the contrary, when the probe and coupling fields are in the nearly counterpropagating geometry, i.e. $\theta \simeq \pi$, the decoherence mechanism is dominated by Doppler effects, and this is why the EIT-related experiments in the counter-propagating geometry are always studied in cold atoms [22].

\section{RESULTS AND DISCUSSIONS}

To demonstrate the availability of the current scheme, we use the experimental data of SL as an input for eqs. 15-17). To highlight the effect of thermal motion of atoms, we consider the case when the probe and cou(14pling beams are arranged with $\theta \simeq \pi$ along the major axis of a cigar-shaped cloud of laser-cooled ${ }^{87} \mathrm{Rb}$ atoms 


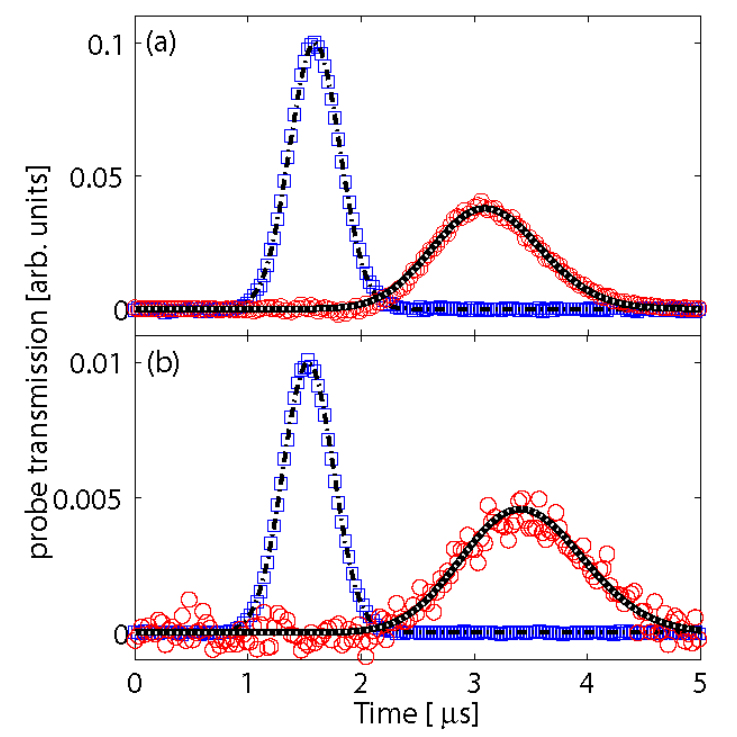

FIG. 2: (Color online) The squares and circles are the experimentally measured intensities of the input and output probe pulses, respectively. The input probe pulses are scaled down by a factor of 0.1 in (a) and 0.01 in (b). The dasheddotted lines represent the numerical simulations of the input probe pulses. The black lines represents the numerical results of the output probe pulse by solving SL in the stationary EIT medium using the effective parameters $\left(\bar{\Omega}_{c}, L \bar{\eta}, \bar{\gamma}\right)=$ $(0.7 \Gamma, 16.13 \Gamma, 0.0143 \Gamma)$ in (a) and $(0.67 \Gamma, 19.98 \Gamma, 0.0227 \Gamma)$ in (b). The gray dotted lines represent the best fitting by using the scheme in [17] with the parameters $\left(T, \Omega_{c}, L \eta\right)=$ $(205 \mu K, 0.71 \Gamma, 16.5 \Gamma)$ in (a) and $(305 \mu K, 0.68 \Gamma, 20.5 \Gamma)$ in (b). Clearly, the black and gray dotted lines completely merge in both (a) and (b).

[21] The experimental values of the coupling Rabi frequency, opacity and relaxation rate of the ground-state coherence (denotes as $\Omega_{c}^{*}, L \eta^{*}$ and $\gamma_{0}^{*}$ ) can be estimated by the method described in [22]. We apply the numerical scheme of [17], which considers all velocity groups of the atoms in the calculations, to determine the temperature $T$ of the atomic medium and get the best fitting of $\Omega_{c}$ and $L \eta$ as the gray dotted lines plotted in Fig.2(a) and (b). To be generic, we consider two independent sets of experimental data of SL with the estimated values $\left(\Omega_{c}^{*}, L \eta^{*}, \gamma_{0}^{*}\right)=(0.687 \Gamma, 15 \Gamma, 0.0005 \Gamma)$ and $(0.69 \Gamma, 20.5 \Gamma, 0.0005 \Gamma)$. The best fitting comes up correspondingly with $\left(T, \Omega_{c}, L \eta\right)=(205 \mu K, 0.71 \Gamma, 16.5 \Gamma)$ and $(305 \mu K, 0.68 \Gamma, 20.5 \Gamma)$. Inserting the above best fitted parameters into eqs. (15)-(17), the effective parameters are found to be $\left(\bar{\Omega}_{c}, L \overline{\bar{\eta}}, \bar{\gamma}\right)=(0.7 \Gamma, 16.13 \Gamma, 0.0143 \Gamma)$ and $(0.67 \Gamma, 19.98 \Gamma, 0.0227 \Gamma)$. Using $\bar{\Omega}_{c}, L \bar{\eta}$ and $\bar{\gamma}$ in eqs.(1)(3), we numerically calculate the output probe pulse as a function of time and find it is in good agreement with the experimental data as the black lines plotted in Fig.2(a) and (b). The validity of eqs.115)-(17) is hence demonstrated.
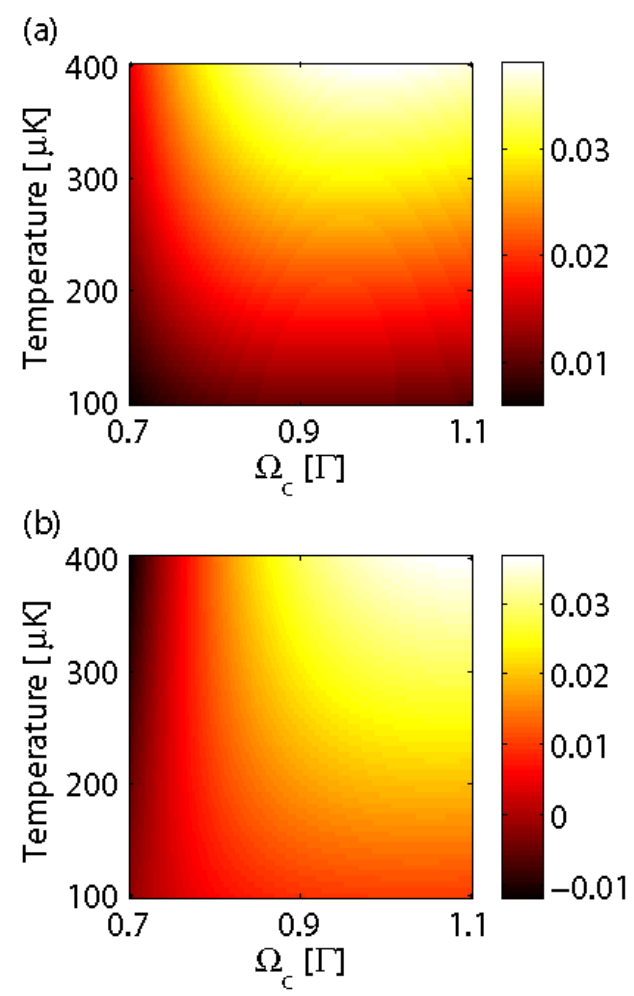

FIG. 3: (Color online) The fractional changes, $\delta \bar{\Omega}_{c}=$ $\left(\Omega_{c}-\bar{\Omega}_{c}\right) / \Omega_{c}$ and $\delta \bar{\eta}=(\eta-\bar{\eta}) / \eta$ are plotted in (a) and (b) as functions of temperatures and $\Omega_{c}$ with $\theta=\pi$.

The proximity of $\bar{\Omega}_{c}$ and $\bar{\eta}$ to their zero-temperature counterparts can be examined by defining the fractional changes, $\delta \bar{\Omega}_{c}=\left(\Omega_{c}-\bar{\Omega}_{c}\right) / \Omega_{c}$ and $\delta \bar{\eta}=(\eta-\bar{\eta}) / \eta$, which are shown in Fig. 3 as functions of $\Omega_{c}$ and $T$ within the experimentally accessible regime. Obviously, the small values of $\delta \bar{\Omega}_{c}$ and $\delta \bar{\eta}$ shown in Fig.3 indicates that, $\Omega_{c} \approx \bar{\Omega}_{c}$ and $\eta \approx \bar{\eta}$, a conclusion that is consistent with the perturbative results in eqs. (15)-(16). We thus expect that the atomic thermal motion affects the ground-state relaxation rate mostly. In Fig.4, we compare the measured ground-state relaxation rate with the theoretical prediction, eq. 177). Here the measured relaxation rate is determined by fitting the experimental data with the numerical results from eqs.(1)-(3). In Fig.4, the theoretical predictions agree with the measured data very well and we see that the averaged temperatures of our experimental setup of $L \eta^{*}=15 \Gamma$ and $20.5 \Gamma$ are found to be $T=200 \mu \mathrm{K}$ and $330 \mu \mathrm{K}$, respectively.

After demonstrating the validity of eq. (17), we study how the decoherence rate $\bar{\gamma}$ varies with $\Omega_{c}, T$ and $\theta$. Giving $\theta=\pi$, we first depict $\bar{\gamma}$ as a function of $\Omega_{c}$ and $T$ in Fig.5. Here we extend the ranges of $\Omega_{c}$ and $T$ so that the conditions of stationary light pulses and those using the counter-propagating beam geometry with lasercooled $\mathrm{Rb}$ atoms can be included. We see that $\bar{\gamma}$ decays 


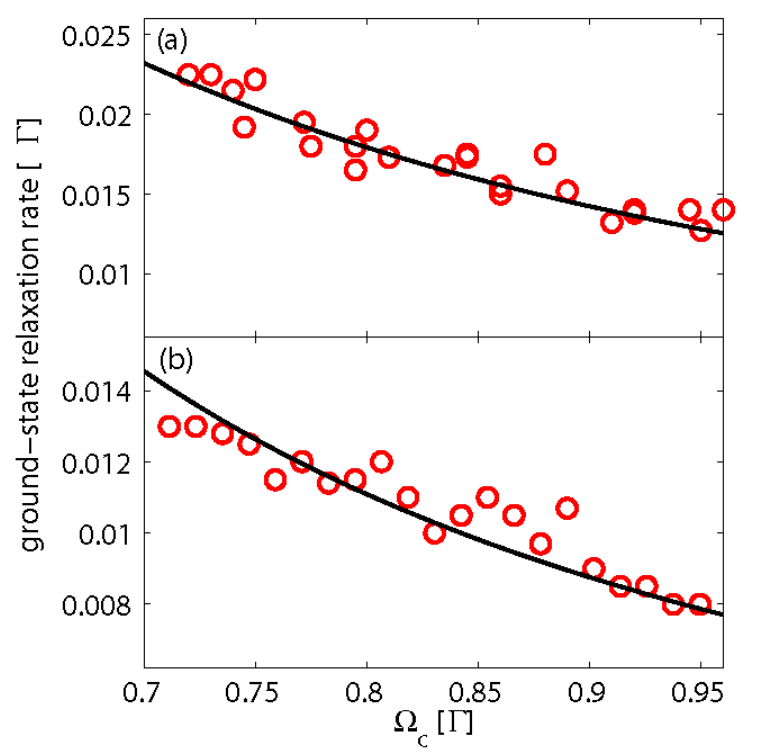

FIG. 4: (Color online) The comparisons between the measured $\gamma$ and the theoretically predicted $\bar{\gamma}$ for $L \eta^{*}=20.5 \Gamma$, $15 \Gamma$ in (a) and (b), respectively. The circles are the measured data and the solid lines are the theoretical predictions at $T=330 \mu \mathrm{K}, 200 \mu \mathrm{K}$ in (a) and (b). The corresponding temperatures of the measured data in (a) range from $290 \mu \mathrm{K}$ to $370 \mu \mathrm{K}$ while those in (b) range from $175 \mu \mathrm{K}$ to $230 \mu \mathrm{K}$.

with increasing intensity of the coupling field as shown in Fig.5. This is because the width of the transparency window depends on the intensity of the coupling field, and an increasing $\Omega_{c}$ can lower the absorption of the probe pulse, so that $\bar{\gamma}$ would become smaller. The influence of the orientation of the coupling beam and the effect of Doppler width on $\bar{\gamma}$ is investigated by plotting $\bar{\gamma}$ as a function of $\theta$ and $k_{p} v_{s} / \Omega_{c}^{2}$ in Figs.6-7, which were obtained based on the numerical calculations of eqs. 12 - (14). The conditions of most experiments utilizing small separation angle between two propagating beams in lase-cooled atoms are met in the ranges, $1^{\circ} \leq \theta \leq 21^{\circ}, 0.01 \Gamma^{-1} \leq k_{p} v_{s} / \Omega_{c}^{2} \leq$ $\Gamma^{-1}$, as shown in Fig.6. On the other hand, those experiments utilizing the nearly co-propagating beam geometry for hot atoms are mostly achieved in the ranges $0.1^{\circ} \leq \theta \leq 2.1^{\circ}, 10 \Gamma^{-1} \leq k_{p} v_{s} / \Omega_{c}^{2} \leq 1000 \Gamma^{-1}$, as shown in Fig.7. Taking our experimental conditions $\left({ }^{87} \mathrm{Rb}\right.$ atoms and $\lambda=780 \mathrm{~nm}$ ) as an example, if $T=100 \mu \mathrm{K}$ then we have $k_{p} v_{s} / \Omega_{c}^{2} \approx 0.03 \Gamma^{-1}$. Figures $5-7$ thus provide useful information to determine the relaxation rate for those EIT-related experiments in which the Dopplerbroadening cannot be ignored.

\section{CONCLUDING REMARKS}

In conclusion, we have theoretically studied the effects of atomic thermal motion on the dynamics of SL in a

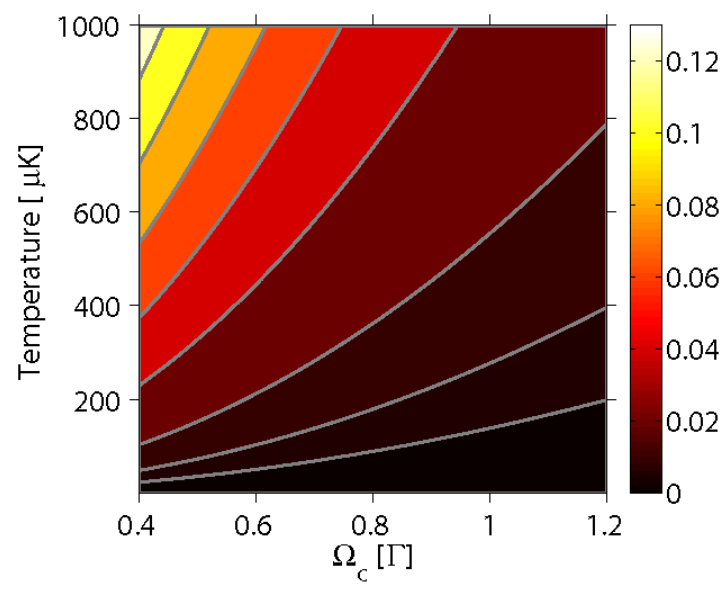

FIG. 5: (Color online) Contour plot of $\tilde{\gamma}$ with $\theta=\pi$. The vertical axis ranges from $T=1 \mu \mathrm{K}$ to $1000 \mu \mathrm{K}$ and the horizontal axis ranges from $\Omega_{c}=0.4 \Gamma$ to $1.2 \Gamma$. The values of the contour lines are from 0.12 (the upper left), 0.1, 0.08, 0.06, $0.04,0.02,0.01,0.005$ (the lower right).

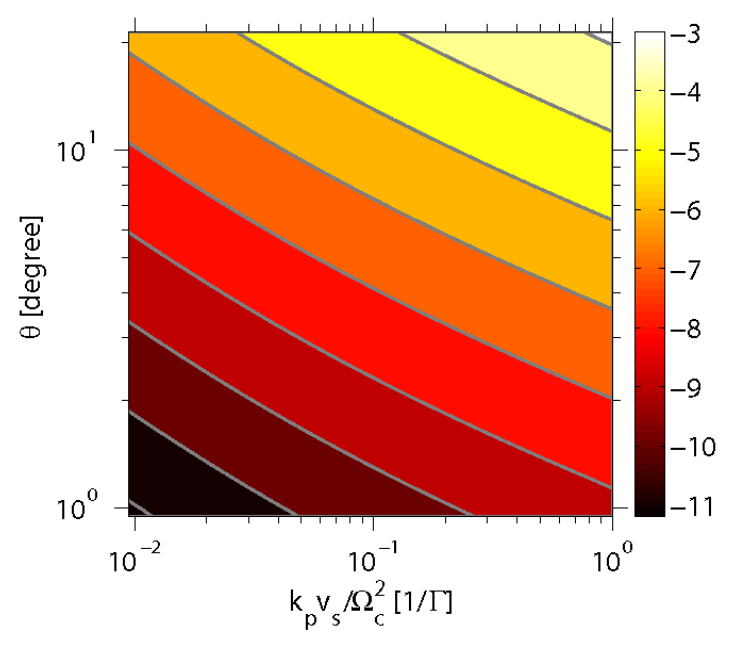

FIG. 6: (Color online) The plot of $\tilde{\gamma}$ as a function of $\theta$ and $k_{p} v_{s} / \Omega_{c}^{2}$ in logarithm scale. The vertical axis ranges from $\theta=1^{\circ}$ to $21^{\circ}$ and the horizontal axis ranges from $k_{p} v_{s} / \Omega_{c}^{2}=$ $0.01 \Gamma^{-1}$ to $\Gamma^{-1}$. The values of the contour lines is from $10^{-3}$ (the upper right) to $10^{-10}$ (the lower left) with a spacing of one order of magnitude.

Doppler-broadened EIT medium. By extending the results in [19], we have obtained the attenuation constant, the delay time and the broadened width of the output probe field at finite temperatures. We also have derived a set of parameters, $\bar{\Omega}_{c}, \bar{\eta}$ and $\bar{\gamma}$, which are temperaturedependent that serve as effective thermal sources in the 


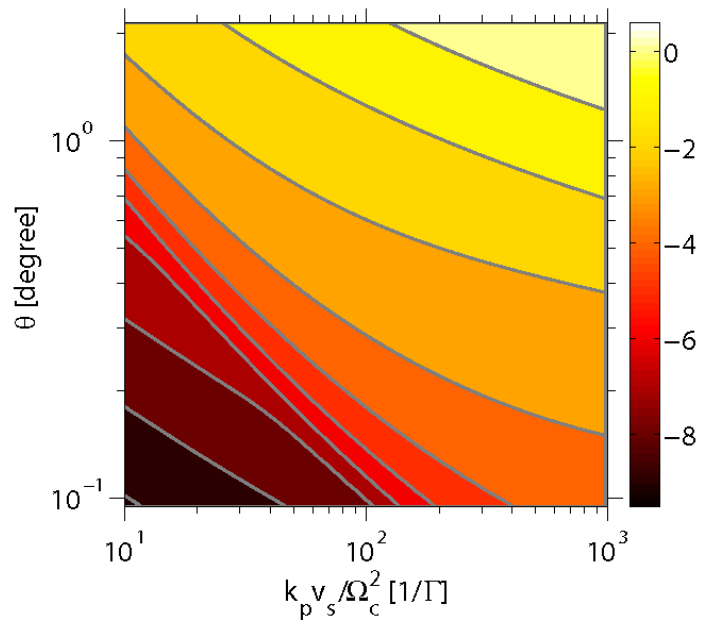

FIG. 7: (Color online) The plot of $\tilde{\gamma}$ as a function of $\theta$ and $k_{p} v_{s} / \Omega_{c}^{2}$. The vertical axis ranges from $\theta=0.1^{\circ}$ to $2.1^{\circ}$ and the horizontal axis ranges from from $k_{p} v_{s} / \Omega_{c}^{2}=10 \Gamma^{-1}$ to $1000 \Gamma^{-1}$. The values of the contour lines are from $10^{0}$ (the upper right) to $10^{-8}$ (the lower left) with a spacing of one order of magnitude. optical Bloch equation for a stationary EIT medium. Our approach provides an approximate and convenient way to determine the relaxation rate of SL instead of appealing to the more complicated multiple-velocity calculations. This effective theory is not only valid for the cold atomic medium but also applicable to the atomic medium at room temperatures.

\section{ACKNOWLEDGEMENTS}

This work is supported by National Science Council, Taiwan, under Grant No. 98-2112-M-018-001-MY2 and No. 98-2628-M-007-004. S.-C. Gou acknowledges the support from National Center for Theoretical Science.
[1] K.-J. Boller, A. Imamoğlu, and S. E. Harris, Phys. Rev. Lett. 66, 2593 (1991).

[2] J. E. Field, K. H. Hahn, and S. E. Harris, Phys. Rev. Lett. 67, 3092 (1991).

[3] D. F. Phillips, M. Fleischhauer, A. Mair, R. L. Walsworth, and M. D. Lukin, Phys. Rev. Lett. 86, 783 (2001).

[4] O. Kocharovskaya, U. Rostovtsev, and M. O. Scully, Phys. Rev. Lett. 86, 628 (2001).

[5] M. Fleischhauer, A. Imamoğlu, and J. P. Marangos, Rev. Mod. Phys. 77, 633 (2005).

[6] S. E. Harris and L. V. Hau, Phys. Rev. Lett. 82, 4611 (1999).

[7] S. E. Harris and Y. Yamamoto, Phys. Rev. Lett. 81, 3611 (1998).

[8] H. Schmidt and A. Imamoğlu, Opt. Lett. 21, 1936 (1996).

[9] M. D. Lukin and A. Imamoğlu, Phys. Rev. Lett. 84, 1419 (2000).

[10] M. O. Scully and M. S. Zubairy, Quantum Optics (Cambridge University Press, Cambridge, U.K., 1997).

[11] L. V. Hau, S. E. Harris, Z. Dutton, and C. H. Behroozi, Nature (Lodon) 397, 594 (1999).

[12] C. Liu, Z. Dutton, C. H. Behroozi, and L. V. Hau, Nature (Lodon) 409, 490 (2001).
[13] D. D. Budker, D. F. Kimball, S. M. Rochester, and V. V. Yashchuk, Phys. Rev. Lett. 83, 1767 (1999).

[14] M. M. Kash, V. A. Sautenkov, A. S. Zibrov, L. Hollberg, G. R. Weich, M. D. Lukin, Y. Rostovtsev, E. S. Fry, and M. O. Scully, Phys. Rev. Lett. 82, 5229 (1999).

[15] A. Javan, O. Kocharovskaya, H. Lee, and M. O. Scully, Phys. Rev. A 66, 013805 (2002).

[16] B. Zhao, Y.-A. Chen, X.-H. Bao, T. Strassel, C.-S. Chuu, X.-M. Jin, J. Schmiedmayer, Z.-S. Yuan, S. Chen and J.W. Pan, M, Nature Phys. 5, 95 (2008).

[17] Jin-Hui Wu, M. Artoni, and G. C. La Rocca, Phys. Rev. A 82, 013807 (2010).

[18] Shih-Wei Su, Yi-Hsin Chen, Shih-Chuan Gou, Tzyy-Leng Horng, and I. A. Yu, Phys. Rev. A 83, 013827 (2011).

[19] Y.-F. Chen, Y.-M. Kao, W.-H. Lin, and I. A. Yu, Phys. Rev. A 74, 063807 (2006).

[20] John David Jackson, Classical Electrodynamics (Wiley; 3 edition, 1998).

[21] Y. W. Lin, H. C. Chou, P. P. Dwivedi, Y.-C. Chen, and I. A. Yu, Opt. Express 16, 3753 (2008).

[22] Y. W. Lin, W. T. Liao, T. Peters, H. C. Chou, J. S. Wang, H. W. Cho, P. C. Kuan and I. A. Yu, Phys. Rev. Lett. 102, 213601 (2009). 